\title{
COMMON FIXED POINTS FOR COMPATIBLE MAPS ON THE UNIT INTERVAL
}

\author{
GERALD JUNGCK
}

(Communicated by James E. West)

\begin{abstract}
Let $g$ be a continuous self-map of the unit interval $I$. It is proved that $g$ has a common fixed point with every continuous function $f: I \rightarrow I$ that is nontrivially compatible with $g$ iff every periodic point of $g$ is a fixed point of $g$.
\end{abstract}

\section{INTRODUCTION}

In 1954 Eldon Dyer asked whether two continuous self-maps $f$ and $g$ of the unit interval that commute $(f g=g f)$ have a common fixed point. Although counterexamples to the conjecture were constructed independently in 1967 by Boyce [1,2] and Huneke [6,7] variants of Dyer's question continue to generate activity $[3,4,12,13]$. One by-product of this activity is the utilization of the commuting map concept as a means of generalization in more abstract settings such as metric spaces. (See [10] for a partial bibliography.)

The desire for more comprehensive results in the context of metric space fixed point theory led to generalizations of the commuting mapping concept per se. One such generalization is the concept of compatible mappings introduced in [10]. As noted in [11], continuous self-maps $f$ and $g$ of a compact metric space $X$ are compatible iff they commute on the set $\{x \in S: f(x)=g(x)\}$ of coincidence points of $f$ and $g$. In this note we return to the unit interval $I=[0,1]$. We prove that a continuous self-map $g$ of $I$ has a common fixed point with every continuous map $f: I \rightarrow I$ that is nontrivially compatible with $g$ iff every periodic point of $g$ is a fixed point of $g$.

\section{NOTATION AND TERMINOLOGY}

The term map will denote a continuous function. As noted above, maps $f, g: I \rightarrow I$ are compatible iff $f g(x)=g f(x)$ for all $x$ in $A=\{x \in I$ : $f(x)=g(x)\}$. If $f$ and $g$ are compatible and $A \neq \varnothing$, we shall say that $f$ and $g$ are nontrivially compatible. So, e.g., if $f(x)=0$ and $g(x)=1$ for $x \in I$, then $f$ and $g$ are trivially compatible maps, but $f$ and $h(x)=x$ are nontrivially compatible on $I$. It is easy to show that if $f, g: I \rightarrow I$ are

Received by the editors January 7, 1989.

1991 Mathematics Subject Classification. Primary 54H25; Secondary 54E45. 
compatible maps such that $f(I) \subseteq g(I)$, then $A \neq \varnothing$ and $f$ and $g$ therefore are nontrivially compatible.

Moreover, suppose that $g: I \rightarrow I$ is a map. $x \in I$ is a periodic point of $g$ iff $g^{k}(x)=x$ for some $k \in N$, the set of positive integers. $P_{g}\left(F_{g}\right)$ will denote the set of periodic (fixed) points of $g$, and $K_{g}$ is the set of all maps $f: I \rightarrow I$ such that $f$ and $g$ are nontrivially compatible.

\section{Results}

The following lemma was stated and proved in [9] for commuting maps; however, the only facts in the proof that required the commutativity of $f$ and $g$ were that the set $A$ of coincidence points of $f$ and $g$ be nonempty and that $f(x), g(x) \in A$ whenever $x \in A$. These facts are obtained if $f$ and $g$ are nontrivially compatible. We can therefore say

Lemma 3.1 [9]. Let $f, g: I \rightarrow I$ be nontrivially compatible maps. If $f$ and $g$ have no common fuxed points, there exist $a, b \in I$ such that

(a) $f(a)=g(a) \geq b>a \geq f(b)=g(b)$,

(b) $f(x) \neq g(x)$ for $x \in(a, b)$.

In the following we shall denote the Intermediate Value Theorem by IVT.

Lemma 3.2. If $g: I \rightarrow I$ is a map and (i) $g^{2}(x)=x$ implies $g(x)=x$, then there exist no $a, b \in I$ such that

$$
g(a) \geq b>a \geq g(b) .
$$

Proof. Suppose there exist $a, b \in I$ for which (*) holds. Since $g$ is continuous, $g$ has a minimum fixed point $d \in(a, b)$. Thus (ii) $g(x)>x$ on $[a, d)$ and $g(d)=d$. Now $g(a) \geq b>d=g(d)$, so that the IVT applied to $g$ on $[a, d]$ yields $z$ in $[a, d)$ such that $g(z)=b$. Consequently, by $(*)$,

$$
g^{2}(z)=g(b) \leq a \leq z .
$$

Keeping in mind that (i) actually assures us that $g(x)=x$ iff $g^{2}(x)=x$, we conclude that $g^{2}(x) \neq x$ for $x \in[a, d)$ by (i) and (ii); consequently, (1) implies that $g^{2}(x)<x$ on $[a, d)$ since $g$ is continuous. But $g^{2}(0) \geq 0$, so there is a largest $r \in[0, a)$ such that $g^{2}(r)=r$. The choice of $r$ and the continuity of $g$ thus imply

$$
g^{2}(x)<x \text { on }(r, d) \text {. }
$$

But $g^{2}(r)=r$ so that $g(r)=r<a<b \leq g(a)$. Apply the IVT to $g$ on $[r, a]$ to obtain $t \in(r, a) \subseteq(r, d)$ such that $g(t)=a$, and hence, $g^{2}(t)=$ $g(a) \geq b>t$. This last inequality is a contradiction of $(2)$.

Lemmas 3.1 and 3.2 combine to give us

Corollary 3.3. If $g: I \rightarrow I$ is a map such that $g^{2}(x)=x$ implies $g(x)=x$, then $g$ has a common fixed point with each function $f \in K_{g}$.

We need the following consequence of Sarkovskiiı's Theorem. See, e.g., [5, p. 62].

Theorem 3.4. Let $g: I \rightarrow I$ be a map, and let $P_{k}=\left\{x \in I: g^{k}(x)=x\right\}$. If $P_{2} \subseteq P_{1}$, then $P_{k}=P_{1}$ for all $k \in N$.

The preceding result enables us to prove 
Theorem 3.5. If a map $g: I \rightarrow I$ has a common fixed point with every $f \in K_{g}$, then $P_{g}=F_{g}$.

Proof. Since in the notation of Theorem 3.4, $P_{g}=\bigcup\left\{P_{k}: k \in N\right\}$ and $F_{g}=$ $P_{1}$, Theorem 3.4 asserts that we can prove that $P_{g}=F_{g}$ by showing that $P_{2} \subseteq P_{1}$. To this end we suppose there exists $a \in P_{2}-P_{1}$. Then $g^{2}(a)=a$ and $g(a) \neq a$. We assume, without loss of generality, that $g(a)>a$ and construct a function $f \in K_{g}$, which produces the desired contradiction.

Consider the interval $[a, g(a)]$. Because of the continuity of $g$, we can let $c$ be the maximum element of $[a, g(a)]$ such that $g(c)=g(a)$ Since $g(g(a))=a<g(a)$, it follows that $c<g(a)$. But $g(g(a))=a$, so we can choose a minimum $d \in(c, g(a)]$ such that $g(d)=a$. Thus $a<g(x)<g(a)$ on $(c, d)$.

Define $f: I \rightarrow I$ as follows:

$$
\begin{aligned}
f(x) & =g(a) \text { if } 0 \leq x \leq c \text { (in particular, } f(a)=g(a)) ; \\
f(c+h) & = \begin{cases}\max \{g(c+h)-h, a\} & \text { if } 0 \leq h \leq(d-c) / 2, \\
\max \{g(c+h)-((d-c)-h), a\} & \text { if }(d-c) / 2 \leq h \leq d-c ;\end{cases} \\
f(x) & =a \text { if } d \leq x \leq 1, \text { (thus, } f(g(a))=a) .
\end{aligned}
$$

Now $f$ is continuous since $g$ is. Moreover, $f$ has no fixed points on $[0, c] \cup[d, 1]$. So the fixed points of $f$ lie on $(c, d)$. But $f(x) \neq g(x)$ on $(c, d)$ by construction. Thus, $f$ and $g$ have no common fixed points. To see that $f$ is indeed a counterexample, we have yet to show that $f$ and $g$ are nontrivially compatible. Since $f(a)=g(a)$, we need only verify that $f$ and $g$ commute on their points of coincidence. Now suppose that $f(x)=g(x)$; then $x \in[0, c]$ or $x \in[d, 1]$. If $x \in[0, c]$ then $f(x)=g(x)=g(a)=f(a)$, so $g f(x)=g^{2}(a)=a$ and $f g(x)=f g(a)=a$; i.e., $g f(x)=f g(x)$. If $x \in[d, 1]$ then $f(x)=g(x)=a$, so $g f(x)=g(a)$ and $f g(x)=f(a)=g(a)$; i.e., $f g(x)=g f(x)$. In any event, $f(x)=g(x)$ implies $f g(x)=g f(x)$, as desired.

Corollary 3.3 and Theorem 3.5 combine to give us the following necessary and sufficient criterion.

Theorem 3.6. A map $g: I \rightarrow I$ has a common fixed point with every function $f \in K_{g}$ iff $P_{g}=F_{g}$.

Corollary 3.7. Suppose that $g: I \rightarrow I$ is continuous on $I$ and differentiable on $(0,1)$. If $g^{\prime}(x) \neq-1$ for $x \in(0,1)$, then $P_{g}=F_{g}$ and $g$ has a common fixed point with every $f \in K_{g}$

Proof. Suppose that $x \in I$ and that $g(x) \neq x$. By the MVT, there is a point $c$ between $x$ and $g(x)$ such that $g(g(x))-g(x)=g^{\prime}(c)(g(x)-x)$. If $g^{2}(x)=x$ we have $x-g(x)=g^{\prime}(c)(g(x)-x)$, or $g^{\prime}(c)=-1$, a contradiction. Thus $g^{2}(x)=x$ implies that $g(x)=x$, so that Corollary 3.3 and Theorem 3.6 yield the conclusion.

It is natural to ask whether at least one fixed point of $g$ assured in Corollary 3.7 must serve as the common fixed point for $g$ and the entire family $K_{g}$. The following example shows that such a point need not exist.

Example 3.8. Let $f, g, h: I \rightarrow I$ be defined by $f(x)=1, g(x)=x^{2}$, and $h(x)=0$ for $x \in I$. It is immediate that $g$ satisfies the hypothesis of Corollary 
3.7 and that $f, h \in K_{g}$, but the only common fixed point of $f(h)$ and $g$ is $1(0)$. (Note that $f$ and $h$ are trivially compatible.)

\section{RETROSPECT}

Consider the functions $f, g, h: I \rightarrow I$ defined below.

Example 4.1. Let $f(x)=(\underline{4 x}$ on $[0,1 / 4]$ and $\underline{1}$ on $[1 / 4,1]), g(x)=(\underline{1}$ on $[0,1 / 4],(\underline{9}=4 x) / 8$ on $[1 / 4,3 / 4]$, and $\underline{x}$ on $[3 / 4,1])$, and let $h(x)=(\underline{x}$ on $[0,3 / 4]$ and $3 / 4$ on $[3 / 4,1])$.

As opposed to Example 3.8, each of the pairs $\{f, g\},\{g, h\}$, and $\{f, h\}$ of Example 4.1 is a nontrivially compatible pair (and we say that the family $\{f, g, h\}$ is nontrivially compatible). For example, $g(x)=h(x)$ implies that $x=3 / 4$, and $g(h(3 / 4))=g(3 / 4)=3 / 4=h(3 / 4)=h(g(3 / 4))$. Now since $g^{2}=g$ and $h^{2}=h$, and-as is easy to show- $f^{2}(x)=x$ implies $f(x)=x$, Theorem 3.4 says that $P_{f}=F_{f}, P_{g}=F_{g}$, and $P_{h}=F_{h}$. Thus we know by Theorem 3.6 or by inspection that each of the pairs in $\{f, g, h\}$ has a common fixed point; but there is no $x \in I$ such that $f(x)=g(x)=h(x)=x$.

The question as to whether commutative families $\mathscr{F}$ of self-maps of $I$ ( $f g=g f$ for all $f, g \in \mathscr{F}$ ) have a common fixed point has been studied by Mitchell [12], Cano [4], and others. In view of the preceding, it is of interest to note that the family $\mathscr{F}$ studied by Cano consisted basically of two classes, one being a commutative class $B$ of maps $f: I \rightarrow I$ such that $P_{f}=F_{f}$. A consequence of the main result (Theorem 1) in [4] is that the class $B$ has a common fixed point. Although the hypothesis of Cano's Theorem 1 required that all functions commute on all of $I$, the proof required commutativity only on the coincidence points and fixed points of functions involved.

\section{REFERENCES}

1. W. M. Boyce, Commuting functions with no common fixed points, Abstract no. 67T-218, Notices Amer. Math. Soc. 14 (1967), 280.

2. __ Commuting functions with no common fixed point, Trans. Amer. Math. Soc. 137 (1969), 77-92.

3. $ـ$, On $\Gamma$-compact maps on an interval and commutativity, Trans. Amer. Math. Soc. 160 (1971), 87-102.

4. J. Cano, Common fixed points for a class of commuting mappings on an interval, Proc. Amer. Math. Soc. 86 (1082), 336-338.

5. Robert 1. Devaney, An introduction to chaotic dynamical systems, Benjamin/Cummings, Menlo Park, CA, 1986.

6. J. P. Huneke, Two counterexamples to a conjecture on commuting continuous functions of the closed unit interval, Abstract no. 67T-2311, Notices Amer. Math. Soc. 14 (1967), 284.

7. On common fixed points of commuting continuous functions on an interval, Trans. Amer. Math. Soc. 139 (1969), 371-381.

8. W. T. Ingram, Concerning periodic points in mappings of continua, Proc. Amer. Math. Soc. 104 (1988), 643-649.

9. G. Jungck, Commuting mappings and common fixed points, Amer. Math. Monthly 73 (1966), 735-738.

10. __ Compatible mappings and common fixed points, Internat. J. Math. Math. Sci. 9 (1986), 771-779.

11. Common fixed points for commuting and compatible maps on compacta, Proc. Amer. Math. Soc. 103 (1988), 977-983. 
12. T. Mitchell, Common fixed points for equicontinuous families of mappings, Proc. Amer. Math. Soc. 33 (1972), 146-150.

13. A. Schwartz, Common periodic points of commuting functions, Michigan Math J. 12 (1965), 353-355.

Department of Mathematics, Bradley University, Peoria, Illinois 61625 